\title{
Clinical outcomes and patterns of severe late toxicity in the era of chemo-radiation for cervical cancer
}

\author{
Uwe Güth · Richard J. Hadwin • Andreas Schötzau • \\ Mary McCormack
}

Received: 31 October 2011/Accepted: 16 December 2011/Published online: 31 December 2011

(C) Springer-Verlag 2011

\begin{abstract}
Background We present a comprehensive analysis of both therapy-induced severe late toxicity and outcome in a cohort of cervical cancer patients following radiation who were treated according to current guidelines and discuss the methodologic problems of systematically reporting these cases. We introduce a revised concept of reporting treatment failure.

Patients and methods The records of 128 cervical cancer patients who received radiation from 2003 to 2008 were reviewed.

Results Thirteen patients (10.2\%) developed severe late toxicity. The combination of heavy smoking and cardiovascular diseases was found to be a significant contributing factor (HR 6.55, 95\% CI $0.99-43.49, p=0.048$ ). Thirty patients $(23.4 \%)$ experienced treatment failure. Of these,
\end{abstract}

U. Güth · R. J. Hadwin

Department of Gynaecological Oncology, North London Gynaecological Cancer Network, University College London Hospital (UCLH), 250 Euston Road, London NW1 2 PG, UK

U. Güth $(\bowtie)$

Department of Gynaecology and Obstetrics, University Hospital Basel, Spitalstrasse 21, 4031 Basel, Switzerland

e-mail: uwe.gueth@unibas.ch

U. Güth

Department of Gynaecology and Obstetrics, Cantonal Hospital Winterthur, Brauerstrasse 15, 8401 Winterthur, Switzerland

A. Schötzau

Schötzau and Simmen Institute for Biomathematics,

Malzgasse 9, 4052 Basel, Switzerland

M. McCormack $(\bowtie)$

Department of Oncology, North London Gynaecological Cancer Network, UCLH, 250 Euston Road, London NW1 2 PG, UK

e-mail: mary.mccormack@uclh.nhs.uk
$12(9.4 \%)$ were defined to have persistent disease, and 18 (14.0\%) developed recurrent disease. Patients with recurrent disease had significantly better survival time $(p<0.001)$. Compared with the persistence subgroup, they had significantly more often multiple sites of relapse (66.7 vs. $8.3 \%, p=0.002)$ and the sites were more often diagnosed outside the pelvis $(70.7$ vs. $7.7 \%, p<0.001)$. Early disease stages (OR 4.46, 95\% CI 1.87-10.63, $p<0.001$ ) and severe late toxicity $(p=0.037)$ were found to be significant factors for an improved disease-free survival. Conclusions A comprehensive depiction of both late therapy-related toxicity and treatment failure requires precise clinical descriptions and analyses of the clinical courses. Our new concept to differentiate treatment failure following radiotherapy in cervical cancer into persistent and recurrent disease permits a clear differentiation between distinct subgroups of patients with regard to prognosis and clinical presentation and will lead to a more precise description of these cases in the future.

Keywords Cervical cancer - Radiotherapy · Chemoradiation · Outcome $\cdot$ Recurrence $\cdot$ Late toxicity

\section{Introduction}

Chemo-radiotherapy (CRT) has been established as the standard of care in the treatment of advanced cervical cancer for the last 10 years [1-5]. The meta-analyses which evaluated CRT trials concluded uniformly that there were insufficient data available to assess the frequency of serious late toxicity associated with this therapeutic approach [1-5]. Data on late toxicity were not recorded for the majority of trials, and where included, substantial information was missing. In addition, the clinical picture of late 
toxicity in individual patients were not described in detail and underreporting may be assumed $[1,4]$. In this paper, we report our centre's outcome data with CRT and present an analysis of late toxicity following CRT and its management.

\section{Materials and methods}

\section{Study cohort}

Between January 2003 and April 2008, 126 newly diagnosed patients with locally advanced cervical cancer, International Federation of Gynecology and Obstetrics (FIGO) stages IB2-IVA, and two patients with pelvic recurrences of early-stage cervical cancer were treated within the North London Gynaecological Cancer Network. Radiotherapy was administered at the Department of Oncology of the University College London Hospital (London, UK). From the entire study cohort of 128 patients, $121(94.5 \%)$ were treated with concomitant CRT. Seven patients $(5.5 \%)$ received radiotherapy only.

The clinicopathologic, treatment and outcome characteristics of the patients included in the study are summarized in Table 1. All patients were initially staged with clinical examination under anaesthesia (EUA, including cystoscopy), magnetic resonance imaging (MRI) of the pelvis and computed tomography (CT) of the abdomen and the chest. In 48 patients (37.5\%), para-aortic lymph nodes were histologically examined after retroperitoneal surgical
Table 1 Clinicopathologic characteristics of 128 patients who had radiation in cervical cancer at the North London Gynaecological Cancer Network: entire study group and the subgroups of patients who had severe late toxicity after radiation, and those who had persistent or recurrent disease

FIGO Fédération International de Gynécologie et d` Obstétrique (International Federation of Gynecology and Obstetrics), $C R$ concomitant CRT

\begin{tabular}{|c|c|c|c|c|}
\hline & $\begin{array}{l}\text { Entire } \\
\text { study } \\
\text { group } \\
n=128\end{array}$ & $\begin{array}{l}\text { Patients with } \\
\text { severe late } \\
\text { toxicity } \\
n=13\end{array}$ & $\begin{array}{l}\text { Patients with } \\
\text { persistent } \\
\text { disease } \\
\text { after } \\
\text { radiation } \\
n=12\end{array}$ & $\begin{array}{l}\text { Patients with } \\
\text { recurrent } \\
\text { disease } \\
\text { after } \\
\text { radiation } \\
n=18\end{array}$ \\
\hline Age (years) & $\begin{array}{l}\text { Mean } 48.5 \\
\text { (range } \\
22-89 \text { ) }\end{array}$ & $\begin{array}{l}\text { Mean 55.0 } \\
\text { (range } \\
27-78)\end{array}$ & $\begin{array}{l}\text { Mean } 50.0 \\
\text { (range } \\
31-81)\end{array}$ & $\begin{array}{c}\text { Mean } 48.5 \\
\text { (range } \\
28-71)\end{array}$ \\
\hline \multicolumn{5}{|l|}{ FIGO stage at presentation (\%) } \\
\hline Stage I & $22(17.2)$ & $1(7.7)$ & - & $2(11.1)$ \\
\hline Stage II & $70(54.7)$ & $6(46.1)$ & $3(25.0)$ & $9(50.0)$ \\
\hline Stage III & $31(24.2)$ & $4(30.8)$ & $7(58.3)$ & $6(33.3)$ \\
\hline Stage IVA & $5(3.9)$ & $2(15.4)$ & $2(16.7)$ & $1(5.6)$ \\
\hline \multicolumn{5}{|l|}{ Paraaortal nodal status (\%) } \\
\hline Histologically positive & $9(7.0)$ & $2(15.4)$ & - & $2(11.1)$ \\
\hline Histologically negative & $39(30.5)$ & $2(15.4)$ & - & $5(27.8)$ \\
\hline No surgical staging & $80(62.5)$ & $9(69.2)$ & $12(100.0)$ & $11(61.1)$ \\
\hline \multicolumn{5}{|l|}{ Histological type (\%): } \\
\hline Squamous-cell carcinoma & $96(75.0)$ & $9(69.2)$ & $11(91.7)$ & $12(66.7)$ \\
\hline Adenocarcinoma & $29(22.7)$ & $4(30.8)$ & $1(8.3)$ & $6(33.3)$ \\
\hline Small-cell carcinoma & $3(2.3)$ & - & - & - \\
\hline Radiotherapy alone & $7(5.5)$ & - & $2(16.7)$ & $1(5.6)$ \\
\hline Concomitant CRT & $121(94.5)$ & $13(100.0)$ & $10(83.3)$ & $17(94.4)$ \\
\hline After radical hysterectomy & $9(7.0)$ & $3(23.1)$ & - & $2(11.1)$ \\
\hline After simple hysterectomy & $5(3.9)$ & $1(23.1)$ & - & - \\
\hline After neoadjuvant chemotherapy & $27(21.1)$ & $1(7.7)$ & $4(33.3)$ & $3(16.7)$ \\
\hline \multicolumn{5}{|l|}{ Concurrent anamnestic conditions (\%) } \\
\hline Heavy smoking ( $>10$ cigarettes/day) & $30(23.4)$ & $6(46.1)$ & $3(25.0)$ & $5(27.8)$ \\
\hline Cardiovascular diseases & $26(20.3)$ & $5(38.5)$ & $3(25.0)$ & $4(22.2)$ \\
\hline \multicolumn{5}{|l|}{ Outcome status in October 2009} \\
\hline Alive, no evidence of disease & $95(74.3)$ & $13(100.0)$ & $1(8.3)$ & $1(5.6)$ \\
\hline $\begin{array}{l}\text { Alive with recurrent/progressive } \\
\text { disease }\end{array}$ & $3(2.3)$ & - & - & $3(16.7)$ \\
\hline Dead, cervical cancer & $25(19.5)$ & - & $11(91.7)$ & $14(77.7)$ \\
\hline Dead, intercurrent illness & $5(3.9)$ & - & - & - \\
\hline
\end{tabular}


exploration. This procedure was introduced into the standard staging protocol in 2005. In nine cases (7.0\%), adjuvant CRT was delivered after radical hysterectomy for apparently early stage disease. Two patients (1.6\%) received CRT as salvage treatment for pelvic recurrences after previous surgery for early-stage disease.

\section{Radiotherapy}

Prior to 2007, radiotherapy was planned using a conventional simulator and information from pelvic MRI scan. Since 2007, all patients undergo CT simulation and threedimensional conformal planning. Radiotherapy was given according to defined protocols as follows:

(a) patients who received primary radiotherapy $(n=112$, 87.5\%): external beam radiation to the pelvis (50.4 Gy/28 fractions/5.5 weeks/10 MV photons) and intracavitary brachytherapy using an intrauterine tube and ovoid system (15 Gy/2 fractions/HDR/point A). Where parametrial invasion was evident, a further boost (5.4 Gy/3 fractions) was delivered to the pelvic side wall.

(b) Patients who underwent adjuvant radiotherapy following hysterectomy $(n=14,10.9 \%)$ : external beam radiation to the pelvis ( $45 \mathrm{~Gy} / 25$ fractions/5 weeks/ $10 \mathrm{MV}$ photons) and vault brachytherapy (13 Gy/2 fractions $/ \mathrm{HDR} / 0.5 \mathrm{~cm}$ from surface of applicator).

(c) Patients who underwent CRT for pelvic recurrence after previous radical hysterectomy $(n=2,1.6 \%)$ : external beam radiation to the pelvis as per (a) and vault brachytherapy as per (b).

No central shielding is used in our protocol.

\section{Concomitant chemotherapy}

One hundred twenty-one patients received concomitant chemotherapy. One hundred and eighteen patients received weekly cisplatin at $40 \mathrm{mg} / \mathrm{m}^{2}$ and three patients received concomitant carboplatin and etoposide for small cell carcinoma. Of these, 96 patients $(82.1 \%)$ received at least five cycles, 14 patients $(12.0 \%)$ received four cycles, three patients $(2.5 \%)$ three cycles and one patient $(0.9 \%)$ two cycles. Twenty-seven patients (21.1\%) received neoadjuvant chemotherapy with weekly carboplatin (AUC 2) and paclitaxel $\left(80 \mathrm{mg} / \mathrm{m}^{2}\right)$ for 6 weeks within a clinical trial [6].

Acute toxicities were managed effectively such that all patients completed their radiation without interruption.

\section{Follow-up}

Three months after completion of treatment, an MRI of the pelvis is performed at our institution to document the response to treatment. Thereafter, patients are clinically evaluated every 3 months for the first 2 years, every 4 months during the third, and then every 6 months. A routine follow-up visit includes a patient history and complete physical examination including inspection of the cervix or the vaginal vault and bimanual pelvic examination. During the follow-up, routine use of imaging techniques is not performed; these are only performed to investigate clinical suggestions of recurrent disease or in the management of therapy-related side effects and toxicities.

At the conclusion of data collection in October 2009, there was complete follow-up information available for all patients included in this study.

\section{Late toxicity}

Late toxicity was defined as that occurring more than 90 days after the first day of radiotherapy. In order to categorize these, we used the Franco-Italian glossary score which describes five grades of increasing severity: grade 0 has no complications, grade 1 mild, grade 2 moderate complications with patients able to maintain normal activity, grade 3 severe complications requiring surgery or causing permanent damage and grade 4 complications resulting in treatment-related death [7].

Type, site and detection method of treatment failure

For patients who had treatment failure, we differentiated between persistent and recurrent disease.

1. Persistence was defined as disease which either progressed during treatment or that which became clinically evident within 6 months of completion of therapy in the irradiated central (i.e., cervix, uterus, vaginal apex/vault) and/or pelvic region.

2. Recurrence was defined as disease at any site, becoming clinically evident $\geq 6$ months after completion of therapy where previous routine post-treatment radiologic examination revealed a complete response. Newly detected distant metastases and extra-pelvic disease, which were not evident at initial presentation, were also considered as recurrent disease, even if they were found within the 6 months period after completion of treatment.

The procedure to distinguish two prognostically different groups of treatment failure on the basis of a 6-month period of recurrence-free survival after completion of therapy refers to a similar and universally established differentiation in ovarian cancer (platinum-resistant and platinumsensitive disease). 
Furthermore, we noted how persistent/recurrent disease presented: (1) symptomatic (symptoms reported by the patient, 2) asymptomatic, found only by physical examination at routine follow-up, (3) asymptomatic, found by radiologic examination only.

Primary, nodal and distant sites of first failure were coded as central $=\mathrm{C}$, pelvic sidewall $=\mathrm{P}$, paraaortic lymph nodes = PA, intraabdominal organs (e.g., peritoneum, liver parenchyma) $=\mathrm{AO}$, supraclavicular lymph nodes $=\mathrm{SC}$, and distant $=\mathrm{D}$.

The study was carried out in accordance with the guidelines of the institutional review board.

\section{Statistical methods}

Using the Kaplan-Meier method, disease-specific survival (DSS) was calculated from the date of diagnosis to the date of death from cervical cancer, or for patients who remained alive, to the date of last follow up. Non-malignancy-related deaths were censored in the statistical analyses according to the same method used for patients who were alive and disease-free. Statistical differences between groups in terms of survival curves were analysed using the log rank test. In order to predict factors contributing to late toxicity and treatment failure, logistic regression was performed; comparisons between nominal parameters were made with the Fisher exact test. A $p$ value $<0.05$ was considered significant. Statistical analyses were performed with $\mathrm{R}$ Development Core Team software, version 2.7.1 (Vienna, Austria).

\section{Results}

The clinicopathologic, treatment and outcome characteristics of the patients included in the study are summarized in Table 1 . At the end of the observation period in October 2009 (median follow-up time of 37 months, range 5-81 months), 25 patients (19.5\%) had died of cervical cancer. Three patients $(2.3 \%)$ were alive with recurrent cervical cancer. 95 patients $(74.3 \%)$ were alive and had no clinical evidence of disease. Five women $(3.9 \%)$ died of intercurrent illness, none of whom had any clinical evidence of recurrent cervical cancer at the time of death.

\section{Late toxicity}

We observed 13 patients $(10.2 \%)$ with grade 3 late toxicity, none of whom had any evidence of recurrence. These cases are summarized in Table 1 and are described in more detail in Table 2. There were no patients with grade 4 toxicity. The median time from completion of CRT to all clinical presentations of severe late toxicity $(n=17)$ was 16.5 months (range 3-72 months); considering only the first presentation in each patient, the median time was 10 months (range 3-31).

With regard to the development of late toxicity, the combination of heavy smoking and cardiovascular comorbidity was found to be a significant contributing factor (HR 6.55, 95\% CI 0.99-43.49, $p=0.048$ ). Age $(p=0.577)$ and disease stage $(p=0.136)$ appeared to have no impact.

\section{Persistent/recurrent disease}

Twelve patients $(9.4 \%)$ had persistent disease and 18 patients $(14.0 \%)$ developed recurrent disease. The patients of both of these subgroups comprise $23.4 \%$ of our study cohort. The characteristics of both subgroups are tabulated in Table 1. Of these 30 patients, $25(83.3 \%)$ died of progressive or metastatic disease and three patients $(10.0 \%)$ were still alive with disease at the conclusion of our data collection. Two patients $(6.6 \%)$ were diagnosed with central disease recurrence only, one diagnosed 4 months and the other 8 months after completion of treatment. Both women were salvaged surgically with total abdominal hysterectomy. At the conclusion of data collection, they were alive with no evidence of disease at 15 and 18 months following the surgical procedures.

Of the 18 patients with recurrent disease, eight patients $(44.4 \%)$ had their conditions diagnosed within 1 year and 17 patients $(94.4 \%)$ within 2 years after the completion of primary therapy.

Table 3 shows timing, detection method and location of first treatment failure. Compared with the persistence subgroup, the patients of the recurrence group had significantly more often multiple sites (66.7 vs. $8.3 \%$, $p=0.002$ ). While the persistent subgroup showed predominantly a uniform treatment failure in the central region, the sites of relapse in the recurrence group were more often diagnosed outside the pelvis (70.7 vs. $7.7 \%$, $p<0.001)$. A similar distribution was found for the dominant site ( 65.0 vs. $8.3 \%, p=0.003)$.

Figure 1 shows the DSS for the entire study cohort and for the patients with persistent and recurrent disease. The 3 -year survival rate was $80.3 \%$ for the entire study group, $22.2 \%$ for the recurrent disease subgroup and $0 \%$ for the persistent disease subgroup (recurrent vs. persistent disease: $p<0.001)$.

Early disease stage (stage I/II vs. stage III/IV: OR 4.46, $95 \%$ CI 1.87 to $10.63, p<0.001)$ and severe late toxicity ( $p=0.037)$ were found to be significant contributing factors towards a better DSS. Age appeared to have no impact $(p=0.485)$. 


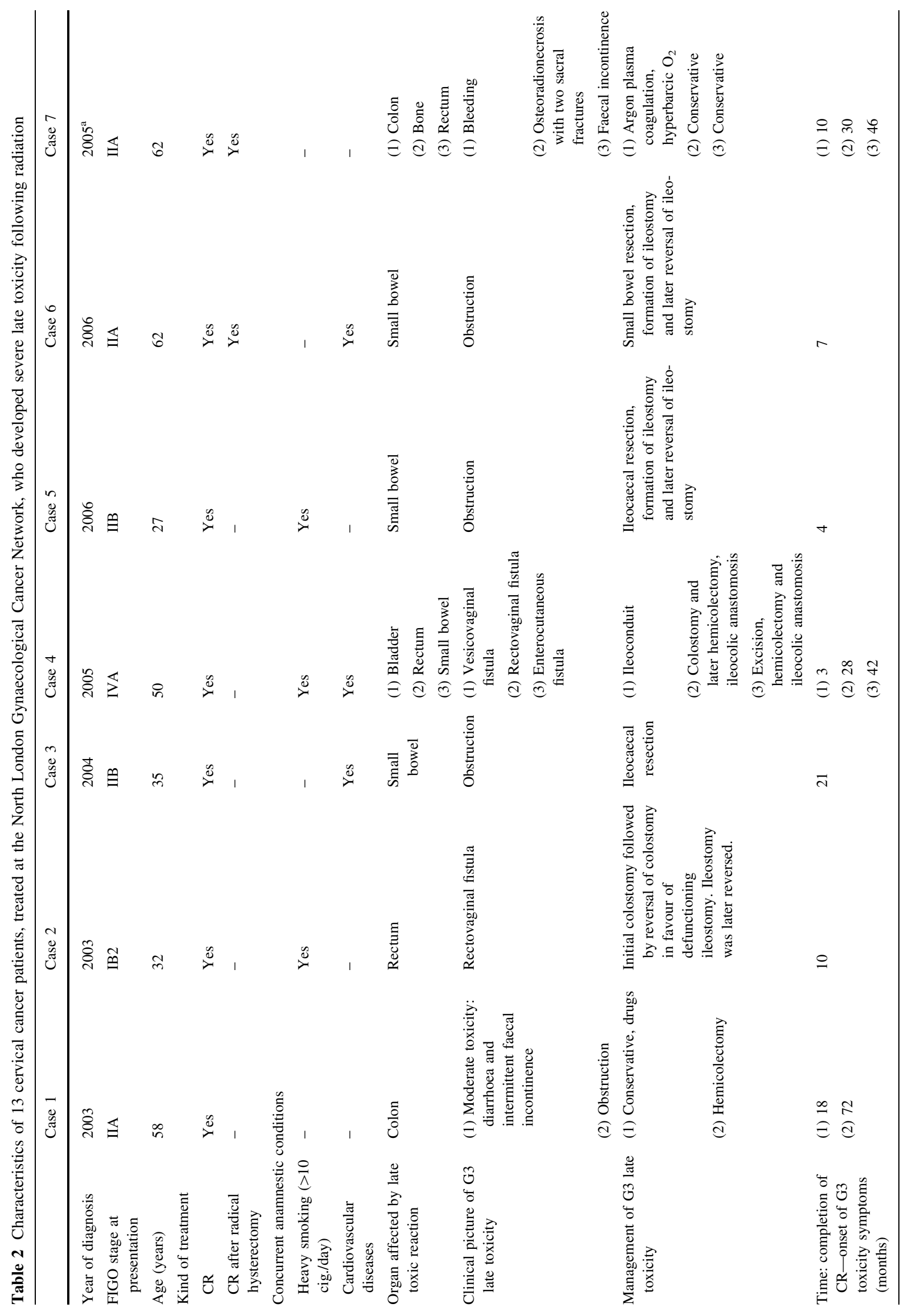




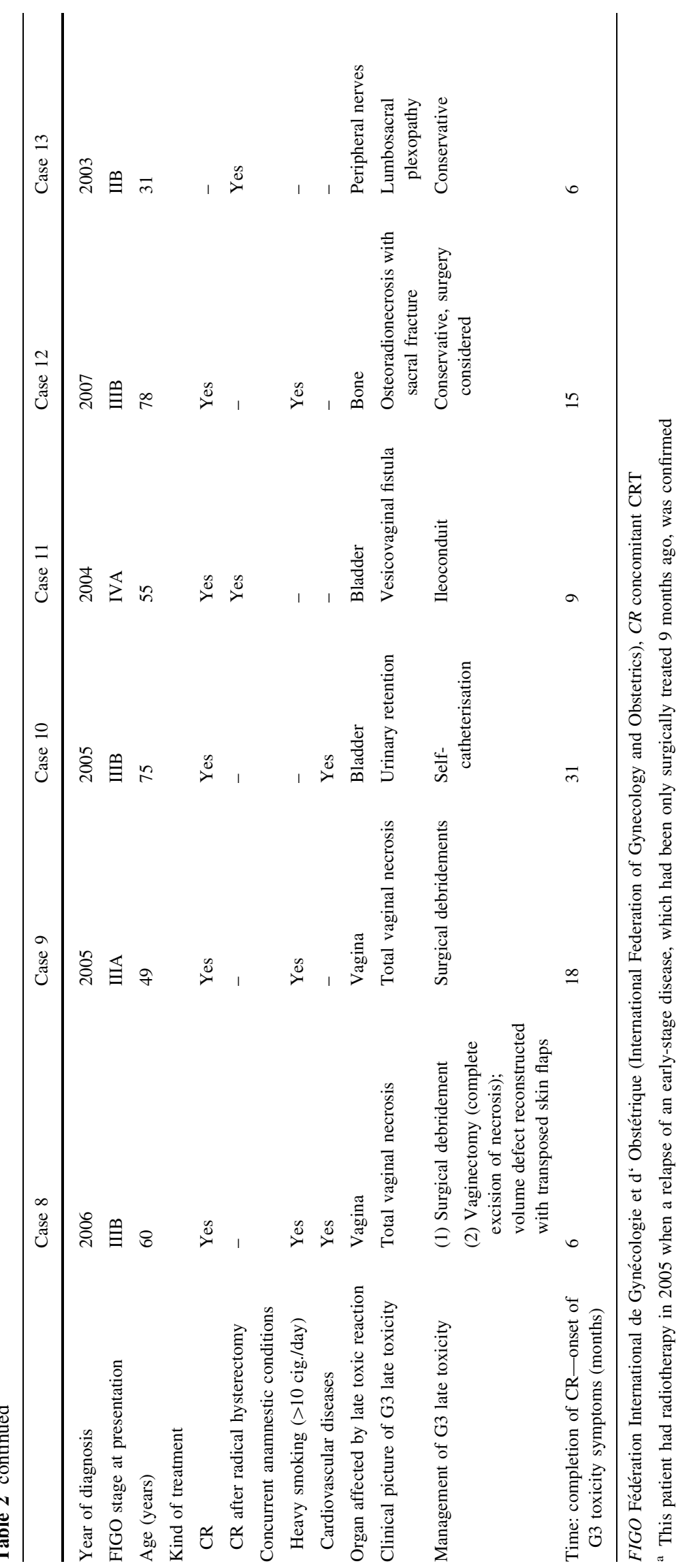


Table 3 Timing, diagnostic method and location of persistent/ recurrent cervical cancer

\begin{tabular}{|c|c|c|}
\hline & $\begin{array}{l}\text { Persistent } \\
\text { disease, } \\
n=12\end{array}$ & $\begin{array}{l}\text { Recurrent } \\
\text { disease, } \\
n=18\end{array}$ \\
\hline $\begin{array}{l}\text { Median time from completion of } \\
\text { treatment to failure (months) }\end{array}$ & $\begin{array}{l}4^{\mathrm{a}} \text { (range } \\
0-5)\end{array}$ & $\begin{array}{l}12 \text { (range } \\
3-49)\end{array}$ \\
\hline $\begin{array}{l}\text { Median follow-up time after } \\
\text { diagnosis of persistent/recurrent } \\
\text { disease (months) }\end{array}$ & 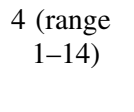 & $\begin{array}{l}8.5 \text { (range } \\
1-22 \text { ) }\end{array}$ \\
\hline \multicolumn{3}{|l|}{ Detection method } \\
\hline Symptomatic & $8(66.7)$ & $12(66.7)$ \\
\hline $\begin{array}{l}\text { Asymptomatic, found by physical } \\
\text { exam }\end{array}$ & - & $2(11.1)$ \\
\hline $\begin{array}{l}\text { Asymptomatic, found by } \\
\text { radiologic exam }\end{array}$ & $4(33.3)$ & $4(22.2)$ \\
\hline Multiple sites at first relapse & $1(8.3)$ & $12(66.7)$ \\
\hline \multicolumn{3}{|l|}{ Sites at first relapse } \\
\hline All sites & 13 & 34 \\
\hline Central & $11(84.6)$ & $7(20.5)$ \\
\hline Pelvic side wall & $1(7.7)$ & $3(8.8)$ \\
\hline Para-aortic lymph nodes & $1(7.7)$ & $9(26.5)$ \\
\hline Intraabdominal organs & - & $2(5.9)$ \\
\hline Supraclavicular lymph nodes & - & $4(11.8)$ \\
\hline Distant sites & - & $9(26.5)$ \\
\hline \multicolumn{3}{|l|}{ Dominant site at first relapse ${ }^{b}$} \\
\hline Central & $11(91.7)$ & $5(25.0)$ \\
\hline Pelvic side wall & - & $2(10.0)$ \\
\hline Para-aortic lymph nodes & $1(8.3)$ & $6(30.0)$ \\
\hline Intraabdominal organs & - & $2(10.0)$ \\
\hline Supraclavicular lymph nodes & - & $2(10.0)$ \\
\hline Distant sites & - & $3(15.0)$ \\
\hline
\end{tabular}

${ }^{a}$ In three cases, a time could not be given because the disease was already progressive under treatment

${ }^{b}$ In two cases, there were two dominant sites clinically equal

\section{Discussion}

Outcomes and patterns of failure

The majority of studies which reported recurrence rates and patterns of recurrence in cervical cancer included patients with early stage disease treated with radical hysterectomy alone, or prior to the adoption of chemoradiation [8, 9]. There are still comparatively few series which have evaluated failure rates in women treated with CRT [10-12]. The Royal College of Radiologists (RCR) in 2010 published the results of a nationwide audit of chemoradiotherapy for locally advanced cervical cancer [13], showing that the addition of chemotherapy to radiotherapy improved survival compared with radiotherapy alone, without an apparent rise in late treatment complications.

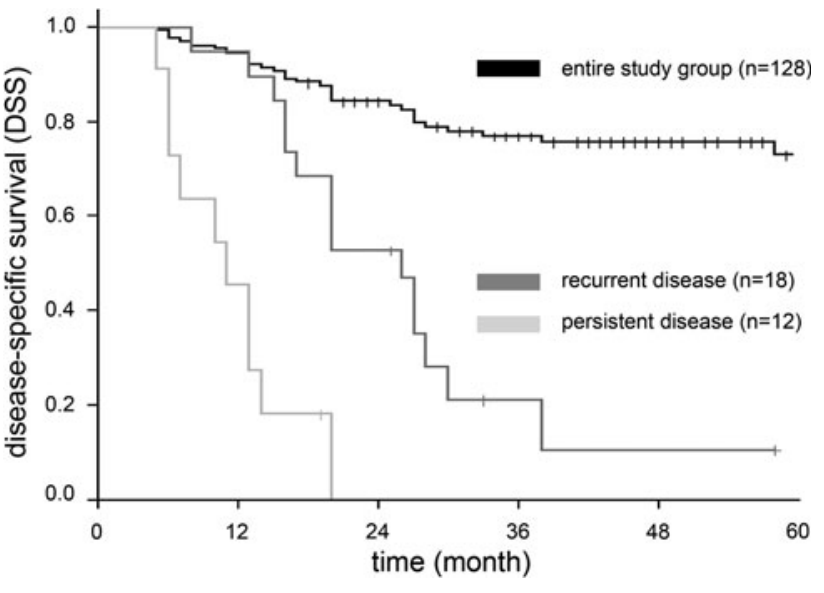

Fig. 1 Disease-specific survival (DSS) of cervical cancer patients who had radiotherapy at the North London Gynaecological Cancer Network. a entire study cohort $(n=128)$, b patients with persistent disease $(n=12)$, c patients with recurrent disease $(n=18)$. Comparison of subgroups B and C: $p<0.001 .+$ censored

The 3-year DSS for patients undergoing chemoradiotherapy were reported as 76, 76 and $54 \%$ for FIGO Stages IB, IIB and IIIB, respectively. The $80.3 \%$ 3-year DSS of our entire cohort compares favourably with these outcomes.

Our data support other similar findings that most of the treatment failures occur within the first 2 years after completion of therapy and most are symptomatic $[8,9]$. In the current published literature of treatment failure in cervical cancer, the corresponding cases have not been further divided. In most of the studies, all cases were summarized under the collective terms "recurrence" or "relapse". In this study we have differentiated between persistent or progressive disease at the end of treatment and true recurrent disease. Our series has shown that there is a dichotomy of clinical course between persistent and recurrent disease. Compared with the persistence subgroup, which had almost exclusively central pelvic disease at the time of treatment failure diagnosis, the recurrence subgroup were more likely to have disease recurrence at multiple sites, often outside the pelvis, and had a significantly better survival.

Similar traditional follow-up protocols with approximately 12-15 visits over a 5-year period are followed universally $[8,9]$. The primary goal of follow-up is to identify recurrent disease in asymptomatic patients at a time when it is amenable to curative salvage therapy. Most authors conclude that this primary goal will not be achieved by routine clinical follow-up in most patients $[8,9]$. Our data support this pessimistic assessment. Clinical examination found asymptomatic recurrences (i.e., not reported by the patients) in only two of the cases with treatment failure. In one case, however, the disease was found in a supraclavicular lymph node and no curative treatment was possible. There was only one patient $(3.3 \%$ 
of all patients with treatment failure, and $5.5 \%$ of the recurrence group) who was diagnosed with a central pelvic recurrence during routine follow-up and she subsequently underwent salvage hysterectomy. In the other case in our series where curative salvage hysterectomy was undertaken, treatment failure was diagnosed at the routine radiologic examination conducted 3 months after completion of CRT (therefore, classified as disease persistence).

Some of the aims for surveillance in cervical cancer patients are potentially better met than the primary goal of recurrence detection, namely to detect and manage complications and toxicities of treatment, to provide patients with psychological support and to collect data for research [8, 9].

Radiation-induced severe late toxicity

In approximately one-half of the published studies regarding CRT, there are no data on late toxicity [1-5]. The reported prevalence varies significantly (1-23\%) [4] with the largest review reporting a generally accepted prevalence of approximately 10\% [14]. The RCR audit also showed an identical crude late Grade 3-4 toxicity rate of $10 \%$ [13]. This heterogeneity between the various studies is not only partly a result of widely different treatment regimens, but also due to a lack of consistency in reporting of these data. Some authors suggest that there may be significant underreporting in terms of late toxicities [1, 4]. Therefore, a meaningful compilation of these cases requires a systematic and detailed description [15]. In some cases, this is challenging since the clinical pictures often overlap and are hard to discriminate from one another. An example of this is the reporting of fistulae. First, we must differentiate between those that are radiation-induced (i.e., in patients who are free of disease) and those occurring in the context of progressive disease. In some cases, there may be a contribution from both treatment effects and recurrent disease. Second, there may be an overlap with a spectrum of clinical pictures and severity, some of which may not fit easily into the current fixed patterns of classification schemes. It is very rare for patients to develop severe late toxicity of a particular organ in isolation. Most patients experience a broad range of mild and moderate sequelae of other organs as well. Currently, there is increasing recognition of the effects of persistent low-grade problems [16]: "the little things that get us down" [17], and these need to be identified. We utilized the Franco-Italian glossary score because it is well recognized and gynaecological cancer specific with detailed descriptions of toxicity patterns [7]. Nonetheless, it can still be difficult to clearly separate between moderate and severe complications. This problem can be illustrated with late toxicity on bowel/ rectum whereby the criteria for severe toxicity are fulfilled in cases where bleeding requires surgery. With the usage of endoscopic methods like Argon Plasma Coagulation treatment for rectal bleeding, the line between when surgery is required and consequently, definition of moderate or severe toxicity becomes blurred. Perhaps more objective parameters are required to distinguish the two as therapeutic modalities for management of toxicities are constantly evolving.

The development of radiotherapy-related late toxicity is likely to be multifactorial. Factors identified as possible contributors include the total dose of radiation and dose per fraction, large radiation field (which implies advanced disease stage), medical comorbidities (particularly cardiovascular disease), smoking, low body mass index and a history of abdominal surgery which may lead to a reduction in the blood supply to the pelvic organs and/or bowel adhesions [14, 15, 18]. A combination of the aforementioned risk factors may potentiate the development of complications.

A potential point of criticism regarding our data could be the fact that we report on different therapy options and not exclusively on patients who had radiochemotherapy as the only treatment (see different therapy forms in Table 1). However, this subgroup does represent the vast majority of our study cohort. We found it particularly interesting to demonstrate patterns of failure and late toxicity, not focusing on a specific treatment but rather from the perspective of the treating clinicians who follow the individual clinical courses. In doing so, we report rare clinical conditions which would otherwise not be reported and have not been so up until now. The colleagues who are interested in the data solely from a homogeneous therapy entity view can easily access this information from our data.

\section{Conclusions}

Differentiating treatment failure of locally advanced cervical cancer into persistent and recurrent disease is useful as this may allow further study of the patient, tumour and treatment factors associated with disease persistence. We hope that our systematic and detailed description of severe radiotherapy-induced late toxicity will help raise awareness and recognition of these sequelae and thus reduce underreporting. The paucity of information regarding serious late toxicity highlights the need for prospective evaluations of outcome, treatment tolerability and quality of life in future trials [1].

Acknowledgments The authors want to thank Adebanke Odujinrin, gynaecological clinical nurse specialist, and Tim Milne, multidisciplinary team coordinator at the UCLH Department of Gynaecological Oncology for their support in obtaining follow-up data of the patients included in this study. 
Conflict of interest The authors declare that there are no financial or personal relationships with other people or organizations that could inappropriately influence the work reported or the conclusions, implications, or opinions stated.

\section{References}

1. Chemoradiotherapy for Cervical Cancer Meta-Analysis Collaboration (2008) Reducing uncertainties about the effects of chemoradiotherapy for cervical cancer: a systematic review and meta-analysis of individual patient data from 18 randomized trials. J Clin Oncol 26:5802-5812

2. Green J, Kirwan J, Tierney J et al. (2005) Concomitant chemotherapy and radiation therapy for cancer of the uterine cervix. Cochrane Database Syst Rev CD002225

3. Green JA, Kirwan JM, Tierney JF et al (2001) Survival and recurrence after concomitant chemotherapy and radiotherapy for cancer of the uterine cervix: a systematic review and metaanalysis. Lancet 358:781-786

4. Kirwan JM, Symonds P, Green JA et al (2003) A systematic review of acute and late toxicity of concomitant chemoradiation for cervical cancer. Radiother Oncol 68:217-226

5. Lukka H, Hirte H, Fyles A et al (2002) Concurrent cisplatinbased chemotherapy plus radiotherapy for cervical cancer-a meta-analysis. Clin Oncol (R Coll Radiol) 14:203-212

6. McCormack M, Ledermann JA, Hall-Craggs MA et al (2009) A phase II study of weekly neoadjuvant chemotherapy followed by radical chemoradiation for locally advanced cervical cancer. J Clin Oncol 27:5586

7. Chassagne D, Sismondi P, Horiot JC et al (1993) A glossary for reporting complications of treatment in gynecological cancers. Radiother Oncol 26:195-202

8. Elit L, Fyles AW, Devries MC et al (2009) Follow-up for women after treatment for cervical cancer: a systematic review. Gynecol Oncol 114:528-535
9. Zanagnolo V, Ming L, Gadducci A et al (2009) Surveillance procedures for patients with cervical carcinoma: a review of the literature. Int J Gynecol Cancer 19:194-201

10. Narayan K, Fisher RJ, Bernshaw D et al (2009) Patterns of failure and prognostic factor analyses in locally advanced cervical cancer patients staged by positron emission tomography and treated with curative intent. Int J Gynecol Cancer 19:912-918

11. Spensley S, Hunter RD, Livsey JE et al (2009) Clinical outcome for chemoradiotherapy in carcinoma of the cervix. Clin Oncol ( $R$ Coll Radiol) 21:49-55

12. Tan LT, Zahra M (2008) Long-term survival and late toxicity after chemoradiotherapy for cervical cancer-the Addenbrooke's experience. Clin Oncol (R Coll Radiol) 20:358-364

13. Vale CL, Tierney JF, Davidson SE, et al. (2002) Substantial improvement in UK cervical cancer survival with chemoradiotherapy: results of a Royal College of Radiologists' audit. Clin Oncol (R Coll Radiol) 22:590-601

14. Eifel PJ, Jhingran A, Bodurka DC et al (2002) Correlation of smoking history and other patient characteristics with major complications of pelvic radiation therapy for cervical cancer. J Clin Oncol 20:3651-3657

15. Güth U, Ella W, Olaitan A et al (2010) Total vaginal necrosis: a representative example of underreporting severe late toxic reaction after oncomitant chemoradiation for cervical cancer. Int $\mathrm{J}$ Gynecol Cancer 20:54-60

16. Maher EJ, Denton A (2008) Survivorship, late effects and cancer of the cervix. Clin Oncol (R Coll Radiol) 20:479-487

17. Vicary P, Johnson M, Maher J (2007) To my oncologist-an open letter from a patient at the end of follow-up. Clin Oncol ( $R$ Coll Radiol) 19:746-747

18. Perez CA, Breaux S, Bedwinek JM et al (1984) Radiation therapy alone in the treatment of carcinoma of the uterine cervix. II. Analysis of complications. Cancer 54:235-246 